\title{
Model assessment of individual tumor control rate and adverse effects in comparing locally advanced cervical cancer treatment using intracavitary with and without interstitial brachytherapy
}

\author{
Hsiang-Chi Kuo, PhD 1,2, Keyur J Mehta, MD',2, Ravindra Yaparpalvi, MSc',2, Viswanathan Shankar, PhD 2,3, \\ William Bodner, MD,2, Madhur Garg, MD,2, Amanda Rivera, MD', Wolfgang A. Tomé, PhD ${ }^{1,2}$, Shalom Kalnicki, MD',2 \\ 'Department of Radiation Oncology, Montefiore Medical Center, New York, ${ }^{2}$ Albert Einstein College of Medicine, New York, \\ ${ }^{3}$ Department of Epidemiology and Population Health, New York, USA
}

\begin{abstract}
Purpose: This study assessed the modeled probability of tumor control and organ at risk toxicities in locally advanced cervical cancer in patients treated by external beam radiation plus brachytherapy using intracavitary combined with interstitial brachytherapy (IC/IS) vs. intracavitary brachytherapy (IC) alone.

Material and methods: Twenty cervical cancer patients with a mean HR-CTV volume of $47.4 \mathrm{~cm}^{3}$ and a mean width of $54 \mathrm{~mm}$ were planned with both IC/IS and IC brachytherapy alone. A probit model was utilized to model 3-year (3-yr) local control rate (LC), 3-yr cancer specific survival rate (CSS), and the adverse effect (AE) of the organ at risk by using a modeled data set from multiple institutions. Modeling results were used to estimate the LC, CSS, and AE of the treatments in this study.

Results: Using the IC/IS technique, an $\mathrm{EQD}_{2}$ increase of $12.3 \mathrm{~Gy}$ to $\mathrm{D}_{90}$ (from $76.1 \mathrm{~Gy}$ to $88.3 \mathrm{~Gy}$ ) of HR-CTV is expected to increase 3-yr LC and 3-yr CSS by 12.5\%, and 11.0\%, respectively. Comparing IC/IS to IC alone, the expected $\mathrm{G}^{+} \mathrm{AE}$ were $7.7 \%$ vs. $7.9 \%$ for the bladder, and $5.9 \%$ vs. $6.8 \%$ for the rectum.

Conclusions: The IC/IS technique improved dose coverage to the HR-CTV without significantly increasing dose to $2 \mathrm{~cm}^{3}$ of the organ at risk (OAR) surrounding it. With different regimens of EBRT combined with BT, IC/IS can be used to increase the probability of LC and CSS, or decrease the risk of AE.
\end{abstract}

Key words: cervical cancer, brachytherapy, tumor control, adverse effect.

\section{Purpose}

External beam radiotherapy (EBRT) with concurrent cisplatin followed by intracavitary brachytherapy (IC BT) has been the standard treatment for locally advanced cervical cancer patients. The evolution of brachytherapy (BT) from 2D to 3D has changed the treatment technique from prescription to Point-A, based on 2D radiographs, to a technique using a $3 \mathrm{D}$ target volume prescription based on CT/MRI images. To adapt to the shape change of the target volume at the time of implant, image guided adaptive brachytherapy (IGABT) was introduced [1]. Specifically, this requires the use of 3D imaging with the applicator in situ at the time of BT. The GEC-ESTRO [2] guidelines for contouring of the high risk clinical target volume (HR-CTV), intermediate risk clinical target volume (IR-CTV), and organs at risk (OAR), as well as dose volume parameters for treatment of locally advanced cervix cancer with IGABT were used.

Evaluation of plan results using dose volume histogram (DVH) parameters requires computerized treatment planning. This development modified the standard loading pattern to conform the target shape or to avoid dose to OAR [3], and facilitated the development of inverse planning processes in gynecologic cases $[4,5,6]$. For bulky and asymmetrical tumors with residual parametrial involvement at the time of BT, the use of intracavitary combined with interstitial brachytherapy (IC/IS BT) $[7,8,9]$ has offered another option to improve the dose coverage for locally advanced cervical cancer. However, its implementation has not been widespread and the clinical results in terms of using IC/IS are yet to be determined. 
We have been routinely using IC/IS with IGABT in the treatment of locally advanced cervical cancer patients with bulky tumors and residual parametrial involvement. This study aims to investigate and assess the clinical advantage of using IC/IS over IC alone in the treatment of locally advanced cervical cancer.

\section{Material and methods}

Twenty patients who underwent CT-guided HDR intracavitary combined with interstitial brachytherapy (IC/IS BT) for cervical cancers (CC) were selected in this retrospective study. Among them, nine were stage IIB and eleven were stage IIIB. Dose was prescribed at 45-54 Gy in 25-28 fractions (four 45 Gy, thirteen 50 Gy, two $50.4 \mathrm{~Gy}$, and one $54 \mathrm{~Gy}$ ) with external beam radiotherapy (EBRT) plus 24-30 Gy (one 3 x 8 Gy, two 4 x 7 Gy, and seventeen $5 \times 6$ Gy) with IC/IS implant.

Nucletron Utrecht applicators (Nucletron, an Elekta company, Elekta AB, Stockholm, Sweden) were used in each implant. The Utrecht applicator is composed of one tandem and two ovoids. Each ovoid has five holes into which needles can be inserted to function as an IC/IS implant. Table 1 summarizes the patient characteristics and the technical data of the IC/IS implants in this study. There were 248 needle inserts performed within a total of

Table 1. Patient characteristics regarding clinical stage at diagnosis, tumor size at brachytherapy

(BT), and the IC/IS implant data in this study

\begin{tabular}{lc} 
Patient characteristics & $\begin{array}{c}\text { Mean } \pm \text { SD } \\
\text { or } n(\%)\end{array}$ \\
\hline Diagnosis & $9(45)$ \\
\hline Stage IIB & $11(55)$ \\
\hline Stage IIIB & $47.4 \pm 19.6 \mathrm{~cm}^{3}$ \\
\hline Tumor size & $5.4 \pm 0.7 \mathrm{~cm}$ \\
\hline Volume & $16(80)$ \\
\hline Width (W) & $4(20)$ \\
\hline W $\geq 5 \mathrm{~cm}$ & 96 \\
\hline W 5 cm & 248 \\
\hline IC/IS Implant & $1.0(0,2.3)^{*}$ \\
\hline Implant no. & $1.2(0.8,2.3)^{*}$ \\
\hline Needle no. & $3.2 \pm 1 \mathrm{~cm}$ \\
\hline Needle & \\
\hline Needle & $3 \pm 0.9 \mathrm{~cm}$ \\
\hline Depth & $57(45.78)$ \\
\hline Depth & $33(12.55)$ \\
\hline Dwell time\% @IC & \\
\hline Dwell time\% @IS & \\
IC/IS - intracavitary combined with interstitial brachytherapy
\end{tabular}

96 implants. On average, each implant had 1.0 inserts via the right ovoid and 1.2 inserts via the left ovoid. The average depth of needle insertion was $3.2 \mathrm{~cm}$ and $3.0 \mathrm{~cm}$ for the right and left ovoids, respectively.

High risk clinical target volume (HR-CTV), intermediate risk clinical target volume (IR-CTV), bladder, rectum, and sigmoid were contoured following the GYN GECESTRO recommendations [2], and the guidelines of CT to MRI target delineation published by Viswanathan et al. [10]. The tumor (HR-CTV) volume and tumor width reported in Table 1 were defined from the CT image of the first BT treatment. Treatment planning was performed with each fraction using the Oncentra ${ }^{\circledR}$ (Nucletron, an Elekta company, Elekta AB, Stockholm, Sweden) treatment planning system. Dose in each BT treatment was prescribed to HR-CTV, with the goal dose being an equivalent dose in 2 Gy fractions $\left(\mathrm{EQD}_{2}\right)$ of $85 \mathrm{~Gy}$, using an a/ $\beta$-ratio of $10 \mathrm{~Gy}$. Optimization was performed manually by limiting the dose constraints of $\mathrm{EQD}_{2}=90$ Gy to $2 \mathrm{~cm}^{3}$ of bladder, and $\mathrm{EQD}_{2}=75 \mathrm{~Gy}$ to both the rectum and sigmoid using an $\alpha / \beta$-ratio of $3 \mathrm{~Gy}$. Dose calculation and reporting were based on the total EQD ${ }_{2}$ of EBRT $+\mathrm{BT}$ using an $\alpha / \beta$ ratio of $10 \mathrm{~Gy}$ for the tumor and an $\alpha / \beta$-ratio of $3 \mathrm{~Gy}$ for the organs at risk (OAR), and a repair half time of $1.5 \mathrm{~h}$.

To compare the difference of the planning results between combined IC/IS implants and IC alone in locally advanced CC, all the original plans (the IC/IS - MO subgroup) were re-optimized by an inverse planning process using a simulated annealing (IPSA) algorithm (the IC/ IS - IPSA sub-group). The inverse planning process kept the OAR dose similar to the original plan by MO while maximizing the coverage to HR-CTV and IR-CTV with the prescription dose. A $2.5 \mathrm{~mm}$ step size was applied for the dwell positions. To prevent small hot spots around an individual dwell positions, a value of 0.3 DTDC (dwell time deviation constraint) was applied to restrict dwell time deviation in each catheter [11]. After optimization was done for an IC/IS plan, the same dose constraints in the IPSA were used to optimize another IC plan (the IC - IPSA sub-group) by inactivating the dwell positions in each interstitial needle. Both IC alone and IC/IS plans using IPSA were finalized by minor manual adjustment of the dwell times to optimize the coverage without increasing the dose to OARs. The purpose of this optimization workflow is to standardize the study. The IPSA method was not clinically utilized in our department.

Plans were compared between IC/IS and IC techniques using the summed $\mathrm{EQD}_{2}$ dose to $90 \%$ volume $\left(D_{90}\right)$ of the HR-CTV, and EQD 2 dose to $2 \mathrm{~cm}^{3}$ volume of the bladder $\left(\mathrm{B}_{2 \mathrm{cc}}\right)$, rectum $\left(\mathrm{R}_{2 \mathrm{cc}}\right)$, and sigmoid $\left(\mathrm{S}_{2 \mathrm{cc}}\right)$. In order to estimate the individual tumor control (TC) and OAR toxicity in this study, long term outcomes based on IGABT technique published by different institutions [12, $13,14,15,16,17,18,19,20,21,22]$ were utilized to model the three-year local tumor control rate (3-yr LC) and threeyear cancer specific survival rate (3-yr CSS). To reduce the variation within the data set, IGABT technique was used as inclusion criteria and data from more than $30 \%$ of patients who were stage IIA or less was excluded [12,13,22]. At the time of this study, only Georg et al. had published large volume-based data modeling the Grade 1-4 toxicity 
of the bladder and rectum $[23,24]$. Since the number of the reported $\mathrm{AE}$ was low, their data was reproduced without further modeling to estimate the Grade 2 and above adverse effect (AE) for each patient in our study.

\section{Probit model}

The long-term outcomes from multiple institutions used in this modeling were summarized in Table 2. The binary outcomes were recorded and displayed as a proportion of the patients that had LC and CSS reported. The proportion data from each institute's report was considered as a quantal data such that the entire data set consists of dose $\left(\mathrm{EQD}_{2}\right.$ to $\mathrm{D}_{90}$ of HR-CTV) and the proportion responding in terms of LC and CSS. A probit model was utilized to examine their association. Probit analysis is a type of regression technique used to analyze binomial response variables. It transforms the sigmoid doseresponse curve to a straight line that can then be analyzed by regression either through least squares (LS) or maximum likelihood (ML). In this study, we utilized the ML method. Proportion data do not have a uniform variance, i.e., as the proportion ( $p$ ) changes (with changing dose), the variance changes. The probit method of linear regression adjusts for this non-constant variance through the application of weights [25]. The variances of the probit regression line can be used to estimate the precision in probit calculation. The $95 \%$ confidence interval (CI) of the probit at each dose level, which was derived from the variance of the probit was calculated and reported. A likelihood-ratio (LR) test was assessed by reporting a $\chi^{2}$ value. Large $\chi^{2}$ lead to a small $p$-value indicating a good model fit. In addition to the variance of the regression line reported by the $95 \% \mathrm{CI}$ and LR test, the area under the curve (AUC) of the receiver operating characteristic (ROC) was used as another figure of merit for comparing alternative model [26].

\section{Modeled outcome assessment between IC/IS and IC technique}

The probit link function is based on the normal cumulated distribution function (CDF). The normal CDF derived after probit analysis was used to assess and predict the individual probability of an outcome from the $\mathrm{EQD}_{2}$ of the HR-CTV using both IC and IC/IS techniques. Evaluated outcomes included the 3-yr LC and 3-yr CSS modeled from the multi-institution data set with above method. The $\mathrm{EQD}_{2}$ to $2 \mathrm{~cm}^{3}$ of bladder and rectum calculated from both IC/IS, and IC plans were used to evaluate the AE of bladder and rectum. They were estimated using the regression curved modeled in Georg's paper [24].

\section{Statistical analysis}

Descriptive summaries of patient and clinical characteristics are presented. Continuous scale variables are pre-

Table 2. Clinical outcomes of locally advanced cervical cancer treated with radiation therapy reported from eight institutions

\begin{tabular}{|c|c|c|c|c|c|c|c|c|}
\hline Factor & GRC [14] & Vien. [15] & STIC [16] & ChiMai [17] & Utrecht [18] & Aarhus [19] & UPMC [20] & UCSD [21] \\
\hline Period & $06-11$ & 01-08 & $05-07$ & 08-11 & $06-08$ & $05-11$ & $07-13$ & 07-14 \\
\hline Imaging & $\mathrm{CT} / \mathrm{MRI}$ & MRI & CT & $\mathrm{CT} / \mathrm{MRI}$ & MRI & MRI & MRI & CT/MRI \\
\hline Patients no & 225 & 156 & 117 & 47 & 46 & 140 & 128 & 76 \\
\hline$\|\mathrm{B} />\| \mathrm{B}$ & $110 / 36$ & $88 / 41$ & $77 / 29$ & $32 / 15$ & $22 / 10$ & $79 / 41$ & $75 / 21$ & $23 / 26$ \\
\hline$W(>5 ;<5)(\mathrm{cm})$ & $-\&$ & $103 ; 53$ & 4.9 & - & 4 & 6 & 5 & - \\
\hline Volume $\left(\mathrm{cm}^{3}\right)$ & - & - & 35.2 & - & $52 / 63$ & - & 31.8 & - \\
\hline IC; IC/IS & - & - & $-; 0$ & - & - & $80 ; 60$ & $121 ; 7$ & $70 ; 0$ \\
\hline$D_{90}\left(G y_{10}\right)$ & $\begin{array}{c}80.4(77.9)^{5} \\
(80 / 71.6)\end{array}$ & $93(96 ; 91)$ & 73.1 & 93 & $84(84 / 76)$ & 91 & 83.2 & 86.3 \\
\hline 3-yr LC (\%) & $\begin{array}{c}86.4(81.9)^{5} \\
(85.5 / 71)\end{array}$ & $\begin{array}{c}95(96 / 86) \\
(98 ; 92)\end{array}$ & 70 & $98(97 / 100)$ & 93 & 91 & 91.6 & 94 \\
\hline $3-y r$ CSS (\%) & $\begin{array}{c}76.1(74.5)^{5} \\
(71 / 83.6)\end{array}$ & $\begin{array}{c}74(84 / 52) \\
(83 ; 70)\end{array}$ & 60 & $\begin{array}{c}85.1 \\
(87.5 / 80)\end{array}$ & $74(69 / 50)$ & 87 & 85.4 & 70 \\
\hline $\mathrm{D}-\mathrm{B}_{2 \mathrm{cc}}\left(\mathrm{Gy} \mathrm{y}_{3}\right)$ & 71.1 & 86 & 69.5 & 88.2 & 83 & $69 / 79$ & 76.5 & 75.3 \\
\hline $\mathrm{D}-\mathrm{R}_{2 \mathrm{cc}}\left(\mathrm{Gy}_{3}\right)$ & 62.1 & 65 & 61 & 69.6 & 66 & $62 / 68$ & 55.9 & 67.5 \\
\hline $\mathrm{D}-\mathrm{S}_{2 \mathrm{Cc}}\left(\mathrm{Gy}_{3}\right)$ & 60.0 & 64 & 58.1 & 72 & 61 & $62 / 73$ & 65.0 & 66.2 \\
\hline $3-y r>G 2^{*}(\%)$ & $4^{2} ; 2.7^{3}$ & $2^{2} ; 3.2^{3}$ & $2.6^{1,2,3}$ & $2^{2} ; 2.1^{3}$ & $2.2^{2} ; 8.7^{3}$ & $1^{2} ; 3^{3}$ & $1^{2,3}$ & $1.5^{3}$ \\
\hline
\end{tabular}

${ }^{*}$ Grade 3 \& 4 toxicity, 1. Gynecologic; 2. Urinary; 3. Gastrointestinal; ${ }^{S}$ value in parenthesis excludes stage bellow IIB; \& data not available; ${ }^{*}$ value in Italic font denotes patient number; the $1^{\text {st }}$ number in (/) denotes value for stage $\| B, 2^{\text {nd }}$ number denotes value for stage $>\| B$; the $1^{\text {st }}$ number in (;) denotes value for $W<5 \mathrm{~cm}$, the $2^{\text {nd }}$ number denotes value for $W>5 \mathrm{~cm}$; IC/IS - intracavitary combined with interstitial brachytherapy; $D_{90}$ - dose to $90 \%$ volume of the HRCTV; $L C$ - local control rate; CSS - cancer specific survival rate; $D-B_{2 c c}$-dose to $2 \mathrm{~cm}^{3}$ of the bladder; $D-R_{2 c c}$-dose to $2 \mathrm{~cm}^{3}$ of the rectum; $D-S_{2 c c}-$ dose to $2 \mathrm{~cm}^{3}$ of the sigmoid; Gy 10 - equivalent dose in 2 Gy fractions an $\alpha / \beta$ ratio of $10 \mathrm{~Gy} \mathrm{~Gy}_{3}$ - equivalent dose in 2 Gy fractions an $\alpha / \beta$ ratio of 3 Gy 
sented as mean (standard deviation) or median (range), as appropriate, while categorical scale variables are presented as frequency count and percentages. A Wilcoxon signed-rank test was used to compare the difference of $\mathrm{EQD}_{2}$, 3-yr LC, 3-yr CSS, and AEs between implant techniques. $P$-value $<0.05$ was considered a statistically significant difference.

\section{Results}

\section{Modeling of treatment outcome}

The reported mean $\mathrm{D}_{90}$ ranges from $\mathrm{EQD}_{2}=73.1 \mathrm{~Gy}$ to $93 \mathrm{~Gy}$, which resulted in 3-yr LC and 3-yr CSS rates of 70\% to $98 \%$ and $60 \%$ to $87.6 \%$, respectively. The fitted curves shown in the left panel of Figure 1 have $\mathrm{ED}_{50}$ values (expected dose with $50 \%$ probability) of $\mathrm{EQD}_{2}=58.5 \mathrm{~Gy}$ and 58.9 Gy for 3-yr LC and 3-yr CSS, respectively. In addition to $\mathrm{ED}_{50}$, the corresponding $\mathrm{ED}_{60}, \mathrm{ED}_{70}, \mathrm{ED}_{80}, \mathrm{ED}_{90}$, and $\mathrm{ED}_{99}$ in $2 \mathrm{~Gy}$ fraction equivalent doses with $95 \%$ confident interval $(95 \% \mathrm{CI})$ were summarized in Table 3.

Tumor control vs. EQD 2

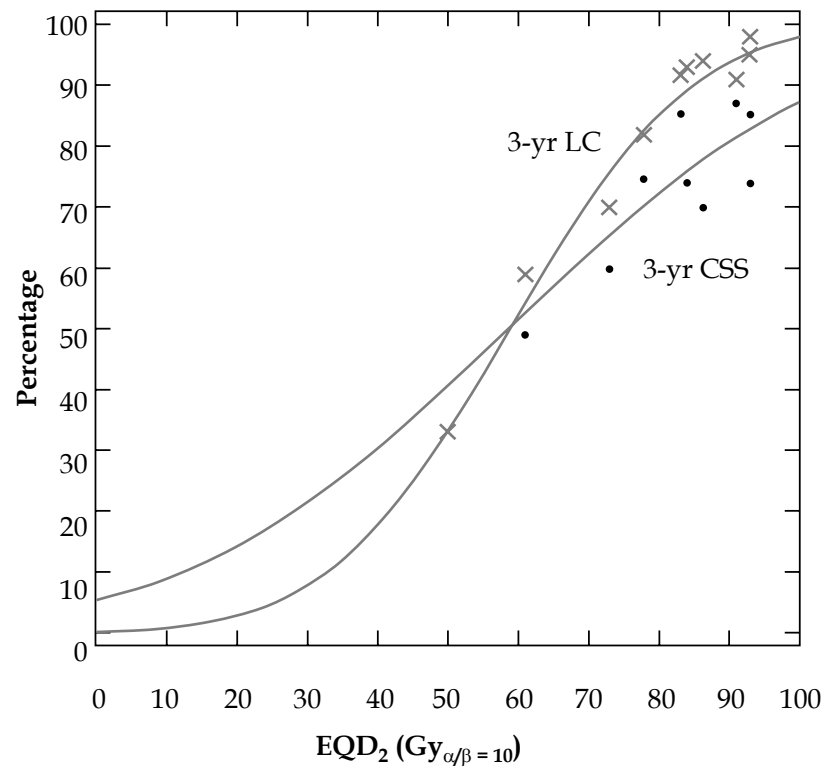

Here $\mathrm{ED}_{\text {level }}$ denotes the expected dose to achieve the specified local control level. Chi-square values were 71.73 $(p<0.0001)$ and $29.85(p<0.0001)$ for LC and CSS, respectively, after LR test. LR test showed good fits of the models for both 3-yr LC and 3-yr-CSS. The AUC values were 0.67 and 0.54 in the model of 3-yr LC and 3-yr CSS, respectively. When comparing the two models, which have the same modeling quantitative data $\left(D_{90}\right)$ but have different end points, the LC model showed a better prediction power than the CSS model. The right panel of Figure 1 reproduced the side effect data published in Georg's paper [24]. Their modeled values of $\mathrm{ED}_{5}, \mathrm{ED}_{10}$, and $\mathrm{ED}_{20}$ along with the 95\% PI were listed in Table 4 for reference. The bladder and rectum dose constraints suggested by GEC-ESTRO and ABS (90 Gy for bladder and 70-75 Gy for rectum) are located between 5-10\% Grade 2 adverse effect in their model, which is clinically acceptable. The regression using the same probit method has goodness of fit values of $0.702(p=0.4)$ and $1.27(p=0.26)$ for bladder and rectum, respectively.

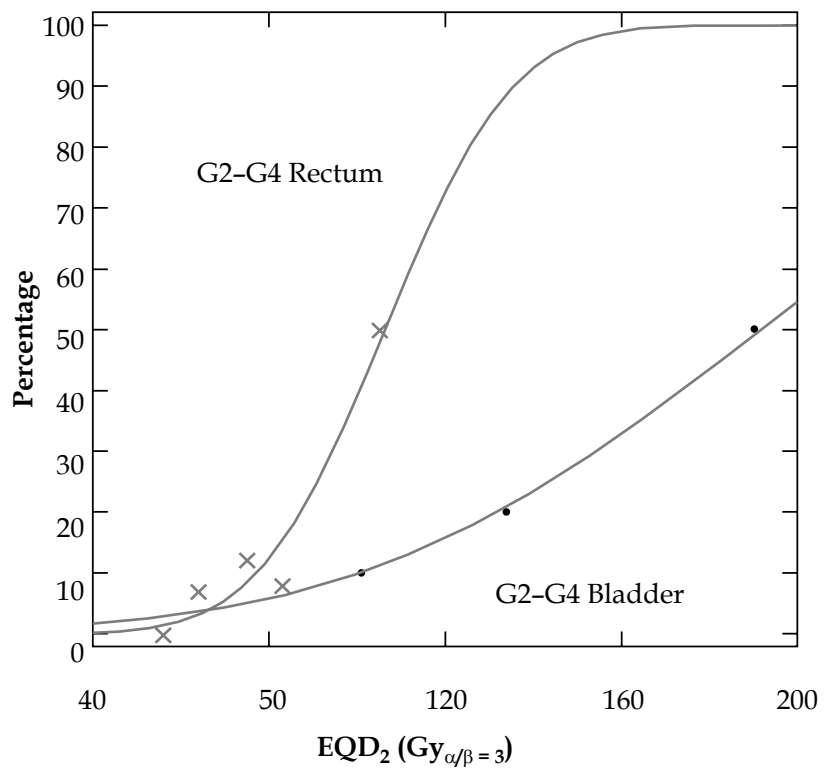

Fig. 1. Dose effect curves depicting 3-yr probability of LC and CSS (left panel: $\times$ - data points for LC model; · data points for CSS model) and Grade 2 (G2) - Grade 4 (G4) risk of rectum and bladder (right panel: $\times$ - data points for rectum AE model; - - data points for bladder AE model)

Table 3. Modeled dose values $\mathrm{ED}_{\text {level }}$ with $95 \% \mathrm{Cl}$ (range in parenthesis) of expected 3-yr level\% LC and 3-yr level\% CSS

\begin{tabular}{lcccccc}
$\mathrm{ED}_{\text {level }}(95 \% \mathrm{Cl})$ & $\mathrm{ED}_{50}$ & $\mathrm{ED}_{60}$ & $\mathrm{ED}_{70}$ & $\mathrm{ED}_{80}$ & $\mathrm{ED}_{90}$ & $\mathrm{ED}_{99}$ \\
\hline 3-yr LC*$(n=903)$ & $58.8(51.6-63.4)$ & $64.0(58.3-67.7)$ & $69.5(65.3-72.4)$ & $76.0(73.1-78.2)$ & $84.9(82.7-87.8)$ & $106.3(100.9-114.7)$ \\
\hline 3-yr-CSS* & $58.6(44.4-65.6)$ & $67.8(58.4-72.6)$ & $77.6(72.8-81.0)$ & $89.2(85.7-94.5)$ & $105.1(98.6-118.5)$ & $143.1(126.9-177.5)$ \\
\hline 3-yr LC $(n=225)$ & $41(-41-56.7)$ & $49.4(-14-62)$ & $58.4(14.6-67.9)$ & $68.9(47-76)$ & $83.5(76.5-103)$ & $118.2(100.5-221)$ \\
\hline 3-yr LC $(n=77)$ & $61(22-70)$ & & $72(57-82)$ & $80(71-97)$ & $92(82-131)$ & \\
\hline 3-yr LC ${ }^{\#} \mathrm{w}>5(n=31)$ & $68(30-78)$ & & $77(67-118)$ & $83(74-137)$ & $91(80-228)$ &
\end{tabular}

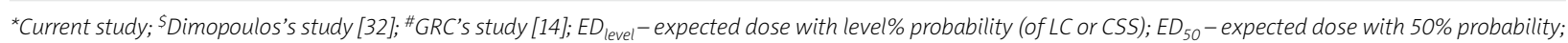
$E D_{60}$-expected dose with $60 \%$ probability; $E D_{70}$ - expected dose with $70 \%$ probability; $E D_{80}$ - expected dose with $80 \%$ probability; $E D_{90}-$ expected dose with $90 \%$ probability; $E D_{99}$ - expected dose with $99 \%$ probability; CSS - cancer specific survival rate; LC - local control rate; GRC - Gustave Roussy Cancer campus 


\section{Dosimetric comparison between IC/IS and IC technique}

Figure 2 displays the distribution of $\mathrm{EQD}_{2}$ to $\mathrm{D}_{90}$ of HR-CTV and the EQD $\mathrm{ED}_{2}$ to $\mathrm{cm}^{3}$ of various OARs using different combinations of EBRT and BT. The median $\mathrm{D}_{90}$ to HR-CTV were 88.3 Gy and 76.1 Gy for the IC/IS and IC techniques, respectively. There was a significant difference in $\mathrm{D}_{90}$ of HR-CTV $(p<0.001)$ when comparing the IC/IS to the IC group. The higher coverage in the IC/IS group only slightly increased the median $\mathrm{EQD}_{2}$ to by $3 \mathrm{~Gy}$ to the sigmoid (73.9 Gy vs. 70.2 Gy), but yielded a 2 Gy reduction for the rectum (71.3 Gy vs. $73.2 \mathrm{~Gy}$ ) and $1 \mathrm{~Gy}$ reduction (89.6 Gy vs. $90.6 \mathrm{~Gy}$ ) for the bladder.

\section{Modeled outcome assessment between IC/IS and IC technique}

Comparing IC/IS and IC alone, the 12.2 Gy (1.01 Gy, $21.5 \mathrm{~Gy}$ ) increase to $\mathrm{D}_{90}$ of HR-CTV in 2 Gy fraction equivalents is expected to increase 3-yr LC and 3-yr CSS by $12.5 \%(0 \%, 20 \%)$, and $11 \%(0.1 \%, 17.6 \%)$, respectively. The expected median (range) of Grade 2 and above AE of the OARs using IC/IS was $7.7 \%(5 \%, 10 \%)$ and $6.3 \%(2 \%$, $23 \%$ ) for bladder and rectum, respectively. The assessed $\mathrm{AE}$ for the bladder was the same between IC/IS and IC techniques. The IC/IS technique decreased assessed $\mathrm{AE}$ in the rectum by $1 \%$. The individual biological model assessments were demonstrated with boxplot distributions in lower left panel (AE) and left panel (LC and CSS) of Figure 2. Compared with IC alone, the IC/IS technique had higher means and narrower ranges in both the 3 -yr $\mathrm{LC}$ and 3-yr CSS. The 1-3 Gy difference in $\mathrm{EQD}_{2}$ between different techniques transforms to $0.2-2 \% \mathrm{AE}$ differences in the range of the results presented in this study.
Table 4. Probability of Grade 2 and above adverse effect according to dose levels reproduced from the study by Georg et al. [24]

\begin{tabular}{lccc}
\multirow{2}{*}{$\begin{array}{l}\text { Dose } \\
\text { volume }\end{array}$} & \multicolumn{2}{c}{$\begin{array}{c}\text { Probability of } \mathrm{EQD}_{2} \text { for G2-G4 side effects (Gy) } \\
\text { for the incident rate shown }(95 \% \mathrm{Cl})\end{array}$} \\
\cline { 2 - 4 } & $5 \%$ & $10 \%$ & $20 \%$ \\
\hline $\mathrm{R}_{2 \mathrm{cc}}$ & $67(30-79)$ & $78(66-110)$ & $90(78-171)$ \\
\hline $\mathrm{B}_{2 c c}$ & $70(0-95)$ & $101(29-137)$ & $134(110-371)$
\end{tabular}

$E Q D_{2}$ - equivalent dose in 2 Gy fractions, $R_{2 c c}-2 \mathrm{~cm}^{3}$ of the rectum, $B_{2 c c}-2 \mathrm{~cm}^{3}$ of the bladder

\section{Discussion}

Interpretation of clinical outcome is complicated and requires data from several clinical trials. If sufficient clinically similar trials were available, results which usually demonstrate the efficacy of a treatment using odds ratio (OR) or relative risk (RR), could be pooled into a metaanalysis to assess the heterogeneity between trials. This could also be used to determine the influence of selected variables (the independent variables) on the effect size (the dependent variable) by using an appropriate meta-regression model [27]. More importantly, the cumulated OR (or RR) determined by meta-analysis concludes a precise measurement of treatment effect. Except the EMBRACE trial, which aims to recruit 600 patients to study the efficacy of the treatment in CC patients utilizing IGABT, no other ongoing trial is available [28]. However, the preliminary outcome of using IGABT from different reports is encouraging in terms of reducing toxicity and increasing local control. Both Dimopoulos and Castelnau-Marchand modeled their LC based on the $\mathrm{EQD}_{2}$ to $\mathrm{D}_{90}$ of the HR-CTV. Their results were listed in Table 3
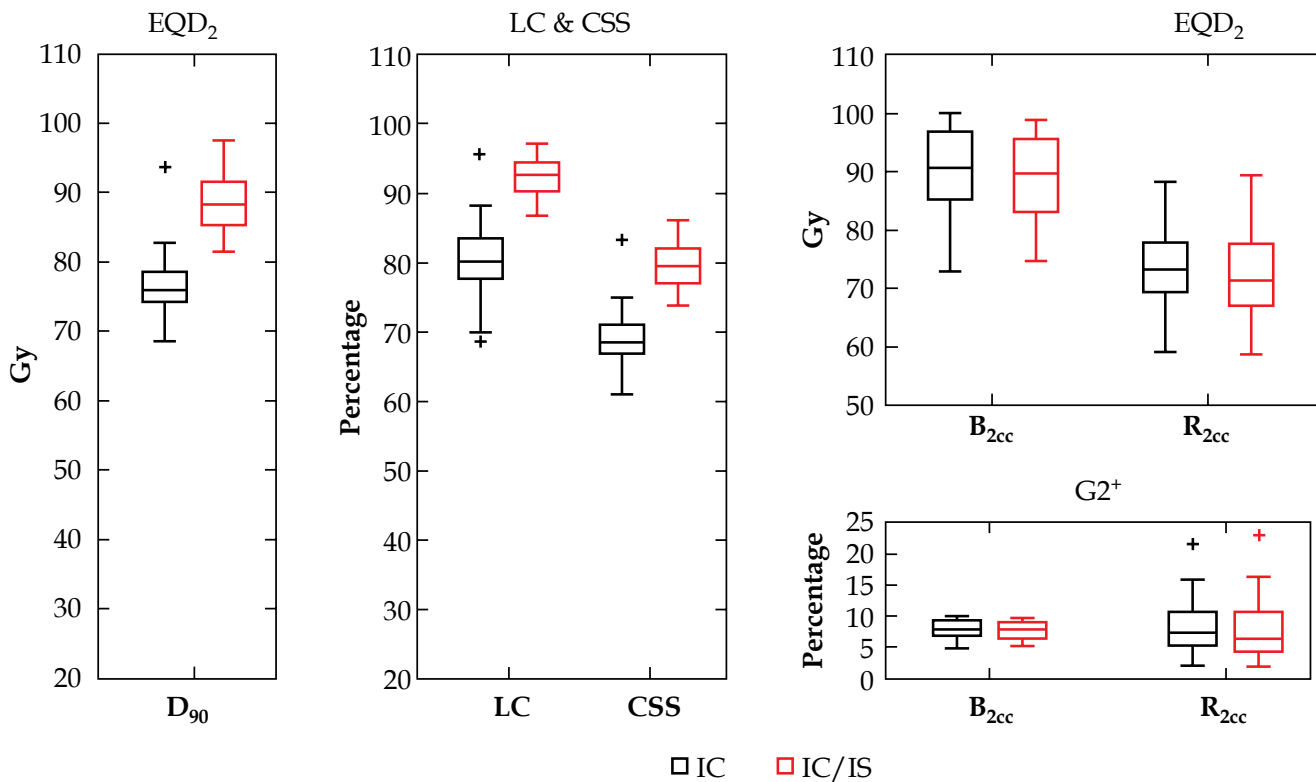

Fig. 2. Left panel: boxplot displaying the $\mathrm{D}_{90}$ distribution (left) and the distribution of the estimated LC, and CSS (right) from the treatment with different combinations of EBRT and BT techniques (IC and IC/IS); right panels: boxplot displaying the $\mathrm{EQD}_{2}$ distribution (up) of the OARs and the estimated Grade 2 and above $\left(\mathrm{G}^{+}\right)$AEs (down) from the treatment with different combination of EBRT and BT techniques (IC and IC/IS) 
for comparison. The $95 \% \mathrm{CI}$ in their reports were large, which could be due to the sample size. Peduzzi's Monte Carlo study suggested a minimum number of 10 events per variable (EPV) to prevent unstable modeling results [29]. The range of data available has great impact on the estimated dose response model [30]. Specifically, the lack of data at the lower dose range can result in a major difference bellow the $\mathrm{ED}_{50}$ position. Treatment outcome also correlates to clinical prognostic factors, such as: stage, histology, volume, hypoxic status, HPP (human papilloma virus pathobiology), age, etc. Other technical factors, which contribute to model variation include delineation of target, imaging modality, and implant technique. Due to the insufficient information available in the literature, this study did not account for the potential uncertainty of the dose response model due to factors other than $D_{90}$ to HR-CTV. It was the major limitation of this study.

The total extent to which models are carried out highly influences the rate of well-predicted absences and the AUC scores [31]. To expand the number of points in the dose range of $50 \mathrm{~Gy}$ to $70 \mathrm{~Gy}$, two additional points (not shown in Table 2) were incorporated in the modeling. The 50 Gy point was read from Figure 2 (33\% LC, estimated to be three patients and was included in LC model only) in the Dimopoulos' paper [32]. Another 61 Gy vs. $56 \% / 50 \%$ (3-yr LC/3-yr CSS) point was adopted from Christi Hospital, which reported 36 CC patients who were not able to be treated with BT [33]. These additional points, which were considered as low or no BT dose contribution, yielded a model that allows for better prediction at a dose range of $50 \mathrm{~Gy}$ to $65 \mathrm{~Gy}$. Due to the dose constraints to OARs, it is difficult to deliver a dose higher than 70 Gy without brachytherapy in CC patients. In two survey reports conducted by Han et al. [34] and Gill et al. [35], the 3-yr CSS of cervix patients treated with brachytherapy vs. no brachytherapy, and with brachytherapy vs. with SBRT/IMRT were $68 \%$ vs. $55 \%$ and $65 \%$ vs. $45 \%$, respectively. An $\mathrm{ED}_{50}$ between 55-60 Gy for both 3-yr LC and 3 -yr CSS is comparable to these clinical results.

The hypothesis in this study was that LC and CSS can be improved by using IC/IS BT for locally advanced CC patients, who normally have a larger tumor at the time of BT and/or higher clinical stage. Several groups have studied the dosimetric differences between using IC/IS and using IC alone using different IC/IS applicators with different study designs. The Netherland Utrecht Medical center used the same Utrecht applicator to study the dosimetric gain of using IC/IS (only in the $2^{\text {nd }}$ fraction of PDR BT) when compared to IC alone in 20 patients [36]. There was an $\mathrm{EQD}_{2}$ of $4.4 \mathrm{~Gy}$ difference in their study. The mean HR-CTV volume at the time BT using IC/IS was $52 \mathrm{~cm}^{3}$. In terms of the number of needles applied and the depth of the needle insert, there were 54 needles (in 20 implants) and a mean of $25 \mathrm{~mm}$ in depth. The mean fraction of the dwell time from the IS component was 19\%. Aarhus University Hospital used the tandem and ring applicator in 24 patients who had a mean HR-CTV volume of $46 \mathrm{~cm}^{3}$ [37]. The mean number of needles implanted was 5.3 and the mean implanted depth was $30 \mathrm{~mm}$. Combined with EBRT dose of 45-50 Gy to pelvis in 25-30 fractions, the dosimetric benefit was from $85.1 \mathrm{~Gy}$ to an
$\mathrm{EQD}_{2}$ of $89.5 \mathrm{~Gy}$. In a historical comparison between IC alone (HR-CTV volume of $33 \mathrm{~cm}^{3}$ ) and IC/IS using tandem and ring (HR-CTV volume of $44 \mathrm{~cm}^{3}$ ) conducted by the Vienna group [7], the $D_{90}$ to the HR-CTV increased from an $\mathrm{EQD}_{2}$ of $87 \mathrm{~Gy}$ to $96 \mathrm{~Gy}$. This difference could be larger if the IC/IS patient group re-optimized with IC only. A multicenter study [38] compared the optimized IC/IS plan vs. optimized IC plan in three CC patients and showed $11.7 \mathrm{~Gy}, 14.8 \mathrm{~Gy}$ and $12.9 \mathrm{~Gy}$ increase to $\mathrm{D}_{90}$ of the HR-CTV. The dosimetric benefit of IC/IS over IC should therefore depend on the tumor size, tumor shape, and the OARs surrounding it.

The dosimetric benefit of using the IC/IS technique for locally advanced CC in this study was statistically significant. It demonstrated an increased $\mathrm{EQD}_{2}$ of $12.3 \mathrm{~Gy}$ when compared to using IC alone. The implantation of the IC/IS technique in this study did not limit the fraction of the dwell time from the interstitial component to be less than $20 \%$ in any single implant as the implantation done by Aarhus University [37] and the Vienna group [7] did. During IPSA optimization, a DTDC (dwell time deviation constraint) value of 0.3 was applied to reduce the deviation between neighboring dwell positions, which decreased the hot spots and heterogeneity of the dose along the implanted needles. The median fraction value of the dwell time from the interstitial component was $33 \%$. This could be the reason why this study has higher $\mathrm{D}_{90}$ increase compared to the results from the other groups $[7,36,37]$.

IC/IS technique geometrically expands the implant dimension compared to IC alone, which is beneficial for larger tumors. According to Dimopoulos's study [32], tumor width $>5 \mathrm{~cm}$ has slightly less LC than width $<5 \mathrm{~cm}$. In a later study by the same group [15], they found that improved LC in tumors $>5 \mathrm{~cm}$ seems to be associated with an increase in 3-yr CSS from $57 \% / 40 \%$ in 1993 $1997 / 1998-2000$ to $70 \%$ in 2001-2008, whereas no change was detectable in tumors $<5 \mathrm{~cm}$. Rijkmans et al. showed a 3 -yr LC rate of $85.9 \%, 89.6 \%$, and $62.4 \%$ for tumor size (at diagnosis) of less than $4 \mathrm{~cm}, 4-6 \mathrm{~cm}$, and greater than $6 \mathrm{~cm}$, respectively [39]. Therefore, a bulkier tumor could receive a better dose coverage using IC/IS technique compared to IC alone, which leads to higher LC and CSS. Tumor stage is a prognostic factor of LC and CSS and is as important as dose coverage. Our modeling results showed that dose is a good predictor of 3-yr LC and 3-yr-CSS for locally advanced CC including stage II and higher stage. However, the larger variation of stage with outcome, as shown in Table 3, implies that the model may work better for stage II but is not as reliable for higher stage. Modeling including both dose and stage as independent explanatory variables is warranted in the future when more outcome data are available.

In addition to increasing the LC and CSS, the evolution of BT from x-ray based implants to IGABT, and of plan evaluation from anatomical points to DVH parameters, has greatly reduced the toxicity of the adjacent OARs. Lindegaard et al. [19] compared 99 patients treated with X-ray based implants to 140 different patients treated with IGABT: the 3-yr CSS increased from $68 \%$ to $87 \%$; the Grade 3 and above combined urological and gastrointestinal $\mathrm{AE}$ were $10 \%$ vs. $4 \%$; the Grade 2 and 
above combined AE were $53 \%$ vs. $35 \%$. In Kang's study [22], the LC was $97 \%$ in the 3D group and was $91 \%$ in the $2 \mathrm{D}$ group. For tumor diameter $>4 \mathrm{~cm}$, the difference of the LC was $98 \%$ vs. $81 \%$. Late rectal breeding (LRB) between the 2D and 3D groups was similar, however, severe LRB rate was significantly lower in the $3 \mathrm{D}$ group. Our study design was to keep similar dose to the OARs (at reasonably level according to GESTRO's constraints) and optimize the dose coverage to HR-CTV. Our results showed minimal dosimetric difference to OAR between IC/IS and IC alone. An insignificant impact on the AE of bladder and rectum was expected because the OARs dose between IC/IS and IC were kept the same. Of note, AEs in our study were assessed using a model from a single institution, and therefore there is potential for higher variation. On the contrary, IC/ IS can be utilized to reduce dose to the OARs, especially in the situation when they are closely surrounding the target. If using IC/IS aims at reducing dose to OARs to demonstrate a large difference in dose to OARs between IC/IS and IC alone, a different model with data compiled from multiple institutions is necessary. In conclusion, clinical outcomes of locally advanced cervical cancer patients from multiple institutions were used to model and predict the individual LC, CSS, and AE of OARs with combined EBRT and BT using IC/ IS or IC alone. The IC/IS technique improved dose coverage to the HR-CTV without significantly increasing dose to $2 \mathrm{~cm}^{3}$ of the OARs surrounding it. This technique potentially can reduce dose to the surrounding OARs at the same time. With different regimens of EBRT combined with BT, IC/IS can be used to increase the probability of $\mathrm{LC}$ and CSS, or decrease the risk of AE.

\section{Disclosure}

Authors report no conflict of interest.

\section{References}

1. Pötter R, Fidarova E, Kirisits C et al. Image-guided adaptive brachytherapy for cervix carcinoma. Clinical Oncol 2008; 20: 426-432.

2. Haie-Meder C, Potter R, Van Limbergen E et al. Recommendations from Gynecological (GYN) GEC-ESTRO Working Group (I): concepts and terms in 3D image based 3D treatment planning in cervix cancer brachytherapy with emphasis on MRI assessment of GTV and CTV. Radiother Oncol 2005; 74: 235-245.

3. Kiristis C, Pötter R, Lang S et al. Dose and volume parameters for MRI-Based treatment planning in intracavitary brachytherapy for cervical cancer. Int Radiat Oncol Biol Phys 2005; 62: 901-911.

4. DeWitt KD, Hsu CJ, Speight J et al. 3D inverse treatment planning for the tandem and ovoid applicator in cervical cancer. Int Radiat Oncol Biol Phys 2005; 63: 1270-1274.

5. Chajon E, Dumas I, Touleimat $M$ et al. Inverse planning approach for 3-D MRI-based pulse-dose rate intracavitary brachytherapy in cervix cancer. Int Radiat Oncol Biol Phys 2007; 69: 955-961.

6. Jamema SV, Kirists C, Mahantshetty U et al. Comparison of DVH parameters and loading patterns of standard loading, manual and inverse optimization for intracavitary brachytherapy on s subset of tandem/ovoid case. Radiother Oncol 2010; 97: 501-506.
7. Kirisits C, Lang S, Dimopoulos J et al. The Vienna applicator for combined intracavitary and interstitial brachytherapy of cervical cancer: design, application, treatment planning, and dosimetric results. Int J Radiat Oncol Biol Phys 2006; 65: 624-630.

8. Jurgenliemk-Schulz IM, Tersteeg RJ, Roesink JM et al. MRIguided treatment-planning optimization in intracavitary or combined intracavitary/ interstitial PDR brachytherapy using tandem ovoid applicators in locally advanced cervical cancer. Radiother Oncol 2009; 93: 322-330.

9. Tan PW, Koh VY, Tang JI. Outpatient combined intracavitary and interstitial cervical brachytherapy: barriers and solutions to implementation of successful programe - a single institutional experience. J Contemp Brachytherapy 2015; 7: 259-263.

10. Viswanathan AN, Dimopoulos J, Kirisits C et al. Computed tomography versus magnetic resonance imaging-based contouring in cervical cancer brachytherapy: results of a perspective trial and preliminary guidelines for standardized contours. Int Radiat Oncol Biol Phys 2007; 68: 491-498.

11. Smith RL, Panettieri V, Lancaster $C$ et al. The influence of the dwell time deviation constraint (DTDC) parameter on dosimetry with IPSA optimization for HDR prostate brachytherapy. Australas Phys Eng Sci Med 2015; 38: 55-61.

12. Tan LT, Coles CE, Hart C et al. Clinical impact of computed tomography-based image-guided brachytherapy for cervix cancer using Tandem-ring applicator - the Addenbrooke's experience. Clinical Oncol 2009; 21: 175-182.

13. Tan LT, Diane Whitney D, Coles CE. Long term outcome of CT-based image-guided brachytherapy for cervix cancer using the tandem-ring applicator. OMICS J Radiol 2014; S1: 003.

14. Castelnau-Marchand P, Chargari C, Maroun P et al. Clinical outcomes of definitive chemoradiation followed by intracavitary pulsed-dose rate image-guided adaptive brachytherapy in locally advanced cervical cancer. Gynecol Oncol 2015; 139: 288-294.

15. Pötter R, Georg P, Dimopoulos JCA et al. Clinical outcome of protocol based image (MRI) guided adaptive brachytherapy combined with 3D conformal radiotherapy with or without chemotherapy in patients with locally advanced cervical cancer. Radiother Oncol 2011; 100: 116-123.

16. Charra-Brunaud $C$, Harter V, Delannes $M$ et al. Impact of 3D image-based PDR brachytherapy on outcome of patients treated for cervix carcinoma in France: results of the French STIC prospective study. Radiother Oncol 2012; 103: 305-313.

17. Tharavichitkul E, Chakrabandhu S, Wanwilairat $S$ et al. Intermediate-term results of image-guided brachytherapy and high-technology external beam radiotherapy in cervical cancer: Chiang Mai University experience. Gynecol Oncol 2013; 130: 81-85.

18. Nomden CN, de Leeuw AAC, Roesink JM et al. Clinical outcome and dosimetric parameters of chemo-radiation including MRI guided adaptive brachytherapy with tandem-ovoid applicators for cervical cancer patients: A single institution experience. Radiother Oncol 2013; 107: 69-74.

19. Lindegaard JC, Fokdal LU, Nielsen SK et al. MRI-guided adaptive radiotherapy in locally advanced cervical cancer from a Nordic perspective. Acta Oncol 2013; 52: 1510-1519.

20. Gill BS, Kim H, Houser CJ, et al. MRI-guided High-Dose-Rate intracavitary brachytherapy for treatment of cervical cancer: The University of Pittsburgh experience. Int J Radiat Oncol Biol Phys 2015; 91: 540-547.

21. Simpson DR, Scanderbeg DJ, Carmona R et al. Clinical outcomes of computed tomography-based volumetric brachytherapy planning for cervical cancer. Int Radiat Oncol Biol Phys 2015; 93: 150-157.

22. Kang HC, Shin KH, Park SY et al. 3D CT-based high-doserate brachytherapy for cervical cancer: clinical impact on late 
rectal bleeding and local control. Radiother Oncol 2010; 97: 507-513.

23. Georg P, Kirists C, Goldner G et al. Correlation of dose-volume parameters, endoscopic and clinical rectal side effects in cervix cancer patients treated with definitive radiotherapy including MRI-based brachytherapy. Radiother Oncol 2009; 91: 173-180.

24. Georg P, Pötter R, Georg D et al. Dose effect relationship for late side effects of the rectum and urinary bladder in magnetic resonance image-guided adaptive cervix cancer brachytherapy. Int J Radiat Oncol Biol Phys 2012; 82: 653-657.

25. Tallarida RJ. Quantal dose-response data: Probit and Logit analysis. In: Drug synergism and dose-effect data analysis. Chapman \& Hall/ CRC, 2000:91-118.

26. Bentzen SM, Constine LS, Deasy JO et al. Quantitative analysis of normal tissue effects in the clinic (QUANTEC): an introduction to the scientific issues. Int J Radiat Oncol Biol Phys 2010; 76: S3-S9.

27. Kokka F, Bryant A, Brockbank E et al. Hysterectomy with radiotherapy or chemotherapy or both for women with locally advanced cervical cancer. Cochrane Database of Systematic Reviews 2015; 4: CD010260.

28. Cihoric N, Tsikkinis A, Miguelez CG et al. Portfolio of prospective clinical trials including brachytherapy: an analysis of ClinicalTrials.gov database. Radiat Oncol 2016; 11: 48.

29. Peduzzi P, Concato J, Kemper E et al. A simulation study of the number of events per variable in logistic regression analysis. J Clin Epidemiol 1996; 49: 1373-1379.

30. Bentzen SM, Tucker SL. Quantifying the position and steepness of radiation dose-response curves. Int Radiat Oncol Biol Phys 1997; 71: 531-542.

31. Lobo JM, Jimenez-Valverde A, Real R. AUC: a misleading measure of the performance of predictive distribution models. Global Ecol Biogeogr 2008; 17: 145-151.

32. Dimopoulos JCA, Pötter R, Georg D et al. Dose-effect relationship for local control of cervical cancer by magnetic resonance image-guided brachytherapy. Radiother Oncol 2009; 93: 311-315.

33. Barraclough LH, Swindell R, Livsey JE et al. External beam boost for cancer of the cervix uteri when intracavitary therapy cannot be performed. Int J Radiat Oncol Biol Phys 2008; 71: 772-778.

34. Han K, Milosevic M, Fyles A et al. Trend in the utilization of Brachytherapy in cervical cancer in the United States. Int J Radiat Oncol Biol Phys 2013; 87: 111-119.

35. Gill BS, Lin JF, Krivak TC et al. National cancer data base analysis of radiation therapy consolidation modality for cervical cancer: the impact of new technological advancements. Int J Radiat Oncol Biol Phys 2014; 90: 1083-1090.

36. Nomden CN, de Leeuw AAC, Moerland MA et al. Clinical use of the Utrecht applicator for combined intracavitary/ interstitial brachytherapy treatment in locally advanced cervical cancer. Int J Radiat Oncol Biol Phys 2012; 82: 1424-1430.

37. Fokdal L, Tanderuo K, Hokland SB et al. Clinical feasibility of combined intracavitary/interstitial brachytherapy in locally advanced cervical cancer employing MRI with a tandem/ ring applicator in situ and virtual preplanning of the interstitial component. Radiother Oncol 2013; 107: 63-68.

38. Nomden CN, de Leeuw AA, Limbergen EV et al. Multicentre treatment study of MRI-guided brachytherapy for cervical cancer: Comparison between tandem-ovoid applicators users. Radiother Oncol 2013; 107: 82-87.

39. Rijkmans EC, Nout RA, Rutten IHHM et al. Improved survival of patients with cervical cancer treated with image-guided brachytherapy compared with conventional brachytherapy. Gynecol Oncol 2014; 135: 231-238. 